\title{
INTEGRAL and Swift observations of IGR J19294+1816 in outburst
}

\author{
E. Bozzo ${ }^{1}$, C. Ferrigno ${ }^{1}$, M. Falanga ${ }^{2}$, and R. Walter ${ }^{1}$ \\ 1 ISDC data center for astrophysics of the University of Geneva, chemin d'Écogia 16, 1290 Versoix, Switzerland \\ e-mail: enrico.bozzo@unige.ch \\ 2 International Space Science Institute (ISSI), Hallerstrasse 6, 3012 Bern, Switzerland
}

Received 15 February 2011 / Accepted 7 May 2011

\section{ABSTRACT}

\begin{abstract}
Context. IGR J19294+1816 was discovered by INTEGRAL in 2009 during a bright X-ray outburst and was classified as a possible Be X-ray binary or supergiant fast X-ray transient.

Aims. On 2010 October 28, the source displayed a second X-ray outburst and a two months-long monitoring with Swift was carried out to follow the evolution of the source X-ray flux during the event.

Methods. We report on the INTEGRAL and Swift observations of the second X-ray outburst observed from IGR J19294+1816.

Results. We detected pulsations in the X-ray emission from the source at $\sim 12.5 \mathrm{~s}$ up to $50 \mathrm{keV}$. The source X-ray flux decreased smoothly during the two months of observation, displaying only marginal spectral changes. Owing to the relatively rapid decay of the source X-ray flux, no significant variations of the source spin period across the event could be measured. This prevented a firm confirmation of the previously suggested orbital period of the source at $117 \mathrm{~d}$. This periodicity was also searched for in archival Swift/BAT data. We detected a marginally significant peak in the periodogram and determined the best period at $116.2 \pm 0.6$ days (estimated chance probability of a spurious detection $1 \%$ ).

Conclusions. The smooth decline of the source X-ray flux across the two months of observations after the onset of the second outburst, together with its relatively low value of the spin period and the absence of remarkable changes in the spectral parameters (i.e., the absorption column density), suggests that IGR J19294+1816 is most likely another member of the Be X-ray binaries discovered by INTEGRAL and not a supergiant fast X-ray transient.
\end{abstract}

Key words. X-rays: binaries - stars: individual: IGR J19294+1816 - stars: neutron - X-rays: stars

\section{Introduction}

IGR J19294+1816 was discovered undergoing a bright X-ray outburst for the first time by INTEGRAL on 2009 March 27 (Turler et al. 2009). On this occasion, IGR J19294+1816 was detected by the IBIS/ISGRI telescope at a significance level of $8.3 \sigma$ in the $20-40 \mathrm{keV}$ energy band. The source was not detected in the $40-80 \mathrm{keV}$ energy band. The IBIS/ISGRI spectrum $(18-50 \mathrm{keV})$ could be reasonably well fitted with a power-law model. The estimated photon index was $\Gamma=4.0 \pm 0.7$ and the corresponding flux $14 \pm 2 \mathrm{mCrab}$ (i.e., $1.1 \times 10^{-10} \mathrm{erg} \mathrm{cm}^{-2} \mathrm{~s}^{-1}$ ).

The analysis of archival Swift observations permitted obtaining a refined position for the source at $\alpha_{\mathrm{J} 2000}=19^{\mathrm{h}} 29^{\mathrm{m}} 55^{\mathrm{s}} .9$ and $\delta_{\mathrm{J} 2000}=18^{\circ} 18^{\prime} 38^{\prime \prime} .4$ (associated uncertainty of 3.5'. at $90 \%$ c.1.), and thus identify the IR counterpart to IGR J19294+1816 as the object 2MASS J19295591+1818382 (Rodriguez et al. 2009b). These data also showed evidence of pulsations at $\sim 12.5 \mathrm{~s}$, later confirmed with RXTE (Strohmayer et al. 2009).

The historical Swift/BAT lightcurve of the source revealed the presence of a modulation with a period of $117 \mathrm{~d}$, which was tentatively interpreted as the orbital period of the system (Krimm et al. 2009; Corbet \& Krimm 2009).

A summary of all these results and an in-depth inspection of all available INTEGRAL observations performed in the direction of the source was provided by Rodriguez et al. (2009a). The authors reported on the discovery of relatively short flares (few thousands of seconds) from this source with INTEGRAL, and concluded that, even though the orbital and spin period of IGR J19294+1816 would nicely place the source in the region of the $P_{\text {spin }}-P_{\text {orb }}$ diagram populated by the Be X-ray binaries, a possible supergiant fast X-ray (SFXTs, see e.g., Walter \& Zurita Heras 2007) nature of this source could not be ruled out.

IGR J19294+1816 was detected undergoing a renewed activity with INTEGRAL on 2010 October 28 (Bozzo et al. 2010; Jenke et al. 2010). Motivated by the previous findings, we triggered a monitoring program of this source with Swift/XRT. This permitted us for the first time to follow in detail the evolution of the source X-ray flux for about two months after the new outburst. In this paper, we report on the results of the INTEGRAL/ISGRI and Swift/XRT observations carried out during the outburst in 2010 and reanalyze all previously available observations performed with these instruments to investigate the real nature of the source. In Sect. 2 we describe the data analysis technique and the results. Our discussion and conclusions are summarized in Sect. 3.

\section{Data analysis}

\subsection{INTEGRAL}

INTEGRAL observations are commonly divided into "science windows" (SCWs), i.e., pointings with typical durations of 2-3 ks. We considered all available SCWs for the IBIS/ISGRI (17-80 keV, Lebrun et al. 2003; Ubertini et al. 2003) telescope that were performed in the direction of IGR J19294+1816 close to the period in which a renewed activity from this source was discovered (see Sect. 1). These comprised observations of the region around GRS1915+105 in satellite revolutions 980, 982, 985,986 , and 988. A complete log of the observations is provided in Table 1 . The source was always outside the field of 


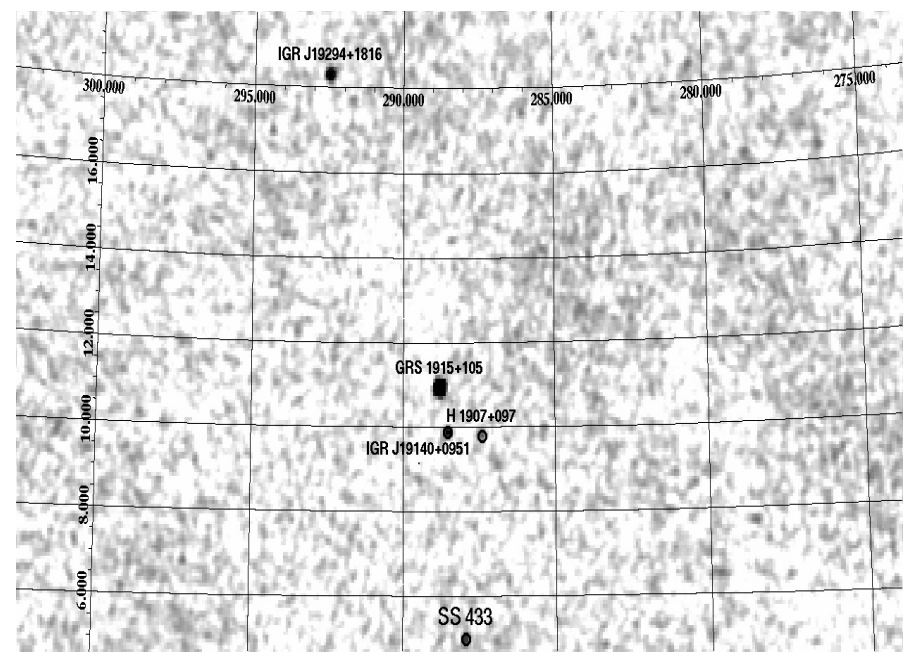

Fig. 1. Mosaic of the IBIS/ISGRI FOV around IGR J19294+1816 realized by using all available SCWs in the direction of the source during satellite revolution 982 (20-50 keV energy range).

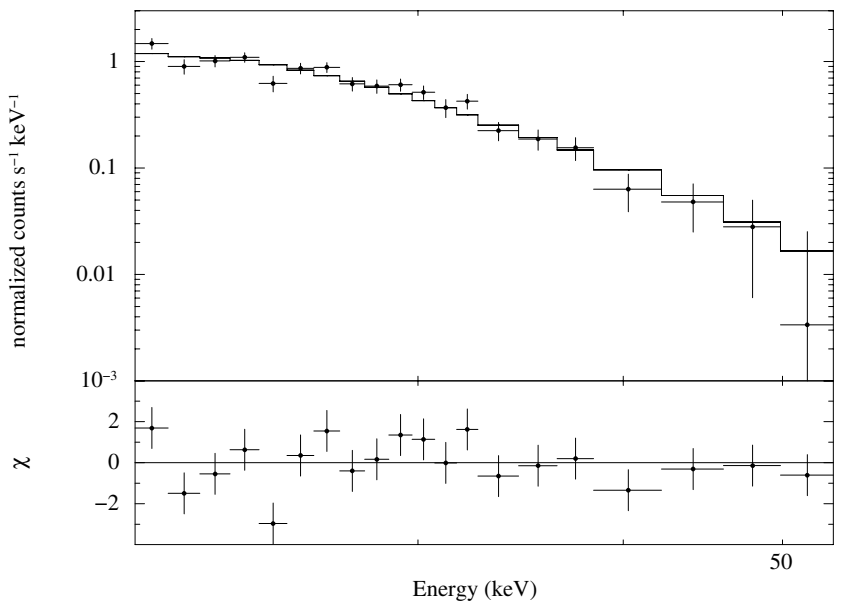

Fig. 2. IBIS/ISGRI spectrum of IGR J19294+1816 extracted from the observations in Rev. 982. The best fit is obtained with a simple powerlaw model and is shown in the figure with a continuous line. The residuals from this fit are reported in the bottom panel.

view (FOV) of the lower energy telescope JEM-X on-board INTEGRAL (Lund et al. 2003). All INTEGRAL data were analyzed using version 9.0 of the OSA software distributed by the ISDC (Courvoisier et al. 2003).

IGR J19294+1816 was detected in rev. 982 at a significance level of $27.3 \sigma$ in the $20-50 \mathrm{keV}$ energy band (this energy band was chosen to maximize the $\mathrm{S} / \mathrm{N}$, see Bozzo et al. 2010). An image of the IBIS/ISGRI FOV around IGR J19294+1816 is shown in Fig. 1. Given the relatively high detection significance, we rebinned the IBIS/ISGRI response matrix ${ }^{1}$ to extract a spectrum with 22 energy bins and look for possible spectral features. A reasonably good fit to this spectrum was obtained using a simple power-law model $\left(\chi_{\text {red }}^{2} /\right.$ d.o.f. $\left.=1.4 / 18\right)$. We measured a powerlaw photon index of $\Gamma=4.9 \pm 0.3$ and estimated a source flux of $(5.4 \pm 0.3) \times 10^{-10} \mathrm{erg} / \mathrm{cm}^{2} / \mathrm{s}(20-50 \mathrm{keV})$. The IBIS/ISGRI spectrum of IGR J19294+1816 is shown in Fig. 2 together with the best-fit model. No convincing spectral features emerged from the residuals of this fit.

\footnotetext{
1 See http://isdcul3.unige.ch/Soft/download/osa/ osa_doc/osa_doc-9.0/osa_um_ibis-9.2/.
}

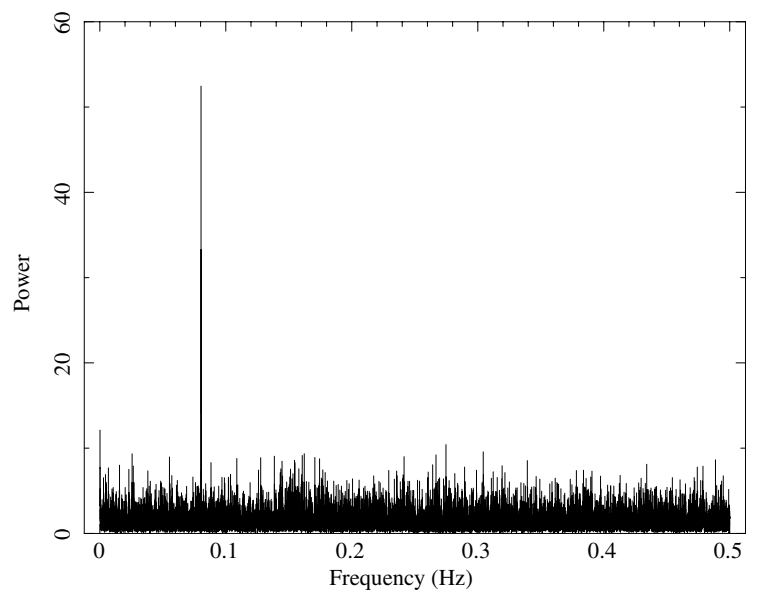

Fig. 3. Power spectrum of the IBIS/ISGRI data of IGR J19294+1816 in Rev. 982. Pulsations at a frequency of $0.080312(6) \mathrm{Hz}$ (error at $90 \%$ c.l. on the last digit) are clearly detected.

We also analyzed the closest INTEGRAL observations performed in the direction of the source before the discovery of its renewed activity. IGR J19294+1816 was in the IBIS/ISGRI FOV from 2010 October 22 at 23:04 to 2010 October 23 at 02:21 (UTC; satellite revolution 980). In this case the source was not detected. We estimated a $3 \sigma$ upper limit on its X-ray flux of $5.1 \mathrm{mCrab}$ (20-50 keV energy band; effective exposure time $22 \mathrm{ks}$ ). This corresponds ${ }^{2}$ to $5 \times 10^{-11} \mathrm{erg} \mathrm{cm}^{-2} \mathrm{~s}^{-1}$.

A similar analysis was also performed on the IBIS/ISGRI mosaic realized by using the available INTEGRAL observations including IGR J19294+1816 in their FOV carried out immediately after the discovery of the renewed activity (satellite Rev. 985, 986, 988). During this period (from 2010 November 6 at 17:46 to 2010 November 18 at 00:26 UTC) the source was not detected and we estimated a $3 \sigma$ upper limit on its X-ray flux in the $20-50 \mathrm{keV}$ energy band of $3.1 \mathrm{mCrab}$ (i.e. roughly $3 \times 10^{-11} \mathrm{erg} \mathrm{cm}^{-2} \mathrm{~s}^{-1}$; effective exposure time $56 \mathrm{ks}$ ).

Pulsations were searched in the INTEGRAL data of rev. 982 by extracting source and background lightcurves with time resolutions of $1 \mathrm{~s}$ applying the method described in Segreto \& Ferrigno (2007, all event times were also corrected to the solar system barycenter). Pulsations were clearly detected in these data (see Fig. 3). We estimated the best pulse frequency at $0.080312(6) \mathrm{Hz}$ (error at $90 \%$ c.l on the last digit) using standard phase-shift techniques (Ferrigno et al. 2007), and measured an rms fractional amplitude of $0.84 \pm 0.06$ (defined according to Eq. (14) in van der Klis 1988). The IBIS/ISGRI pulsed profile of IGR J19294+1816 is shown in Fig. 4.

In order to better compare these results with the previous findings, we also reanalyzed the INTEGRAL data of the previous outburst of IGR J19294+1816 (Rev. 788, see Sect. 1). In this case, we found no clear indication of pulsations in the IBIS/ISGRI periodogram (the source X-ray flux was a factor of $\sim 5$ lower than that in rev. 982). However, an epoch-folding search around the previously know period led to a marginal detection of pulsations. We determined a best pulse frequency of $0.08036(2) \mathrm{Hz}$, and estimated a fractional $\mathrm{rms}$ of $0.55 \pm 0.12$. The IBIS/ISGRI lightcurve of this observation folded at the best determined spin frequency is shown in Fig. 4.

2 The conversion between the source count-rate and $\mathrm{mCrab}$ is carried out by using the IBIS/ISGRI observation of the Crab in Rev. 967. Here the count-rate of the Crab in the $20-50 \mathrm{keV}$ energy band was $168.86 \pm 0.09$ and the corresponding flux $1.0 \times 10^{-8} \mathrm{erg} \mathrm{cm}^{-2} \mathrm{~s}^{-1}$. 

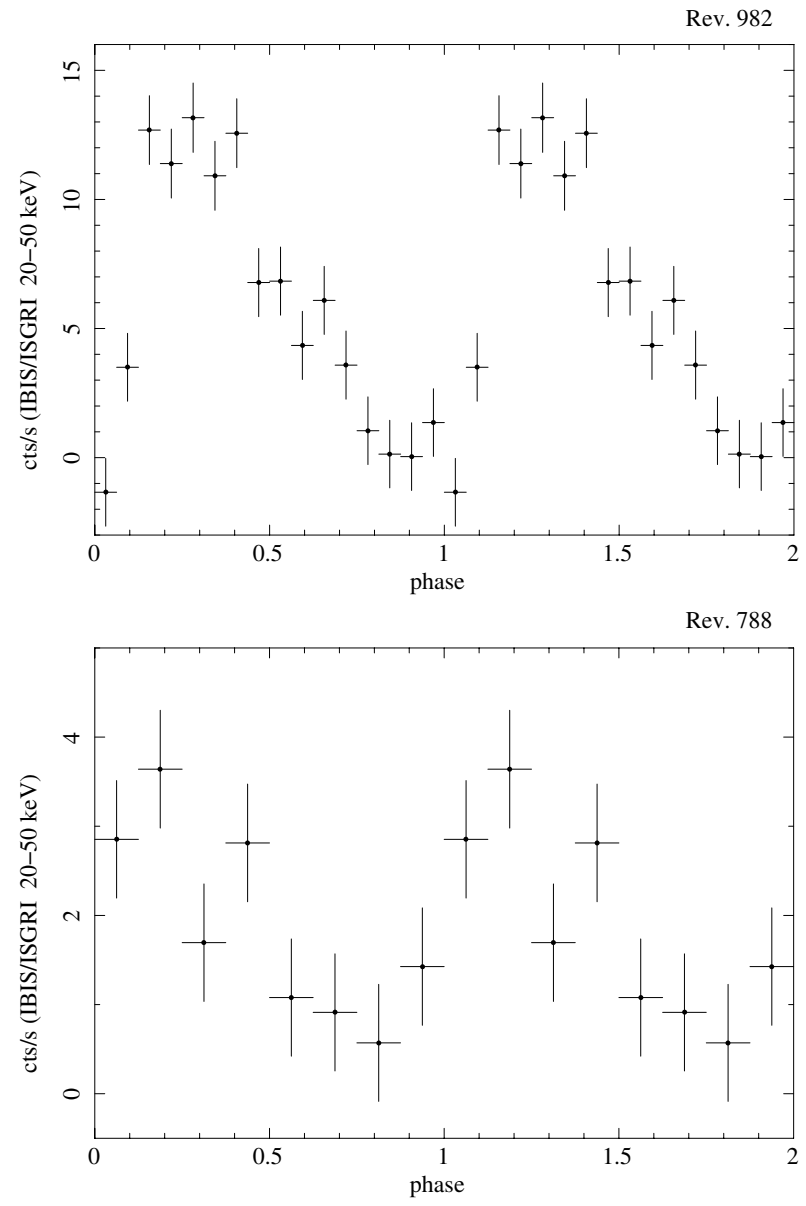

Fig. 4. IBIS/ISGRI lightcurve of IGR J19294+1816 in rev. 982 (top panel) and rev. 788 (bottom panel) folded at the best determined spin frequency. In both cases we used data in the $20-50 \mathrm{keV}$ energy range.

Table 1. INTEGRAL observations log of IGR J19294+1816.

\begin{tabular}{lcccc}
\hline \hline Rev. & $\begin{array}{c}\text { Start time } \\
(\mathrm{MJD})\end{array}$ & $\begin{array}{c}\text { Stop time } \\
(\mathrm{MJD})\end{array}$ & $\begin{array}{c}\mathrm{Exp} \\
(\mathrm{ks})\end{array}$ & $\begin{array}{c}F_{\mathrm{X}} \\
\left(\mathrm{erg} \mathrm{cm}^{-2} \mathrm{~s}^{-1}\right)\end{array}$ \\
\hline 788 & 54917.49852 & 54917.73836 & 12 & $1.1 \times 10^{-10}$ \\
$980^{a}$ & 55491.96168 & 55492.22987 & 22 & $<5.0 \times 10^{-11}$ \\
982 & 55497.76780 & 55498.00752 & 3.4 & $5.4 \times 10^{-10}$ \\
$985,6,8^{a}$ & 55506.74066 & 55518.01843 & 56 & $<3.0 \times 10^{-11}$ \\
\hline
\end{tabular}

Notes. $F_{\mathrm{X}}$ is the flux in the $20-50 \mathrm{keV}$ energy band. EXP indicates the effective exposure time of each observation. ${ }^{(a)} 3 \sigma$ upper limit. Table 1

A $\log$ of all the INTEGRAL observations used is reported in

\subsection{Swift}

Following the detection of a new outburst from IGR J19294+1816, we requested a monitoring observation of the source to follow closely the evolution of its X-ray flux down to the quiescent level. A log of all Swift/XRT observations is provided in Table 2 .

Swift/XRT data were analyzed by using standard procedures (Burrows et al. 2005) and the latest calibration files available. The XRT data were processed with the XRTPIPELINE (v.0.12.6); filtering and screening criteria were applied by using fTools (HEasoft v.6.10). We extracted source and background light

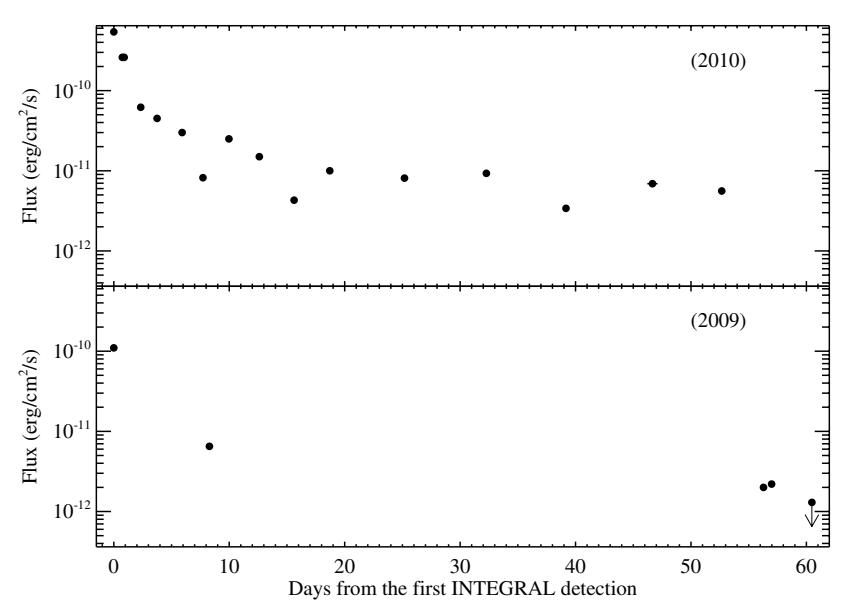

Fig. 5. Lightcurves of the outburst decay of IGR J19294+1816 during the two events that occurred in 2009 (lower panel) and 2010 (upper panel). We did not plot the observations carried out in 2007 because in that case the beginning of the outburst was not caught by any X-ray satellite (see Tables 1 and 2). We scaled the values on the $X$-axis to the first INTEGRAL detection of the source (first point on the left in both panels). This corresponds to 55497.8877 MJD for the upper panel and 54917.6184 MJD for the lower panel.

curves and spectra by selecting event grades of $0-2$ and $0-12$ for the window time and photon counting (PC) mode, respectively. Exposure maps were created through the XRTEXPOMAP task, and we used the latest spectral redistribution matrices in the HEASARC calibration database (v.011). Ancillary response files, accounting for different extraction regions, vignetting and PSF corrections, were generated by using the XRTMKARF task. When required, we corrected PC data for pile-up, and used the XRTLCCORR to account for this correction in the background-subtracted light curves. Source and background event lists were extracted by using the highest possible time resolution for the PC $(2.5 \mathrm{~s})$ and the WT $(0.0018 \mathrm{~s})$ observational modes, and then barycentered by using the BARYCORR tool available within the HEASOFT software package.

From the observations in Table 2 that were characterized by adequate statistics, we extracted source and background spectra and rebinned these spectra to have at least 20 photons per bin to permit $\chi^{2}$ fitting. We derived a mean $\mathrm{X}$-ray flux by fitting these spectra with an absorbed power law model (we used XSPEC v.12.6.0). The spectra extracted during observations characterized by a lower statistic were instead rebinned to have at least five photons per bin and were then fitted by using the $\mathrm{C}$-statistic. For the observations in which less than 100 counts were recorded from the source, we estimated the source count-rate with sosTA (XIMAGE V.4.5.1) and then used this count-rate within weBPIMMs ${ }^{3}$ to derive the X-ray flux (we assumed the same spectral model of the closest observation for which a proper spectral analysis could be carried out; see e.g., Bozzo et al. 2009). All these results are reported in Table 2 and used in Fig. 5 to plot the variation of the source X-ray flux during the decay from the outburst.

Even though the observations were not strictly simultaneous, we performed a broad-band $(0.3-50 \mathrm{keV})$ fit to the source spectrum by combining the IBIS/ISGRI data from rev. 982 and the Swift/XRT data from the observation ID. 00031854001. We used first a simple absorbed blackbody or power-law model, and introduced a normalization constant to take into account both

${ }^{3}$ http://heasarc.nasa.gov/Tools/w3pimms.html 
Table 2. Swift observations log of IGR J19294+1816.

\begin{tabular}{|c|c|c|c|c|c|c|c|c|}
\hline OBS ID & Instr & $\begin{array}{l}\text { Start time } \\
(\mathrm{MJD})\end{array}$ & $\begin{array}{l}\text { Stop time } \\
(\mathrm{MJD})\end{array}$ & $\begin{array}{c}\text { Exp } \\
(\mathrm{s})\end{array}$ & $\begin{array}{c}N_{\mathrm{H}} \\
\left(10^{22} \mathrm{~cm}^{-2}\right) \\
\end{array}$ & $\Gamma$ & $\begin{array}{c}F_{\mathrm{obs}} \\
\left(\mathrm{erg} \mathrm{cm}^{-2} \mathrm{~s}^{-1}\right)\end{array}$ & $\begin{array}{c}\chi_{\text {red }}^{2} / \text { d.o.f. } \\
\text { (C-stat/d.o.f.) }\end{array}$ \\
\hline \multicolumn{9}{|l|}{2007} \\
\hline 00037191001 & XRT/PC & 54443.05208 & 54444.94375 & 7.9 & $4.4_{-0.8}^{+1.1}$ & $0.8_{-0.2}^{+0.3}$ & $3.2 \times 10^{-11}$ & $0.9 / 70$ \\
\hline 00037191002 & XRT/PC & 54447.01389 & 54447.35347 & 3.4 & $3.7_{-1.1}^{+1.3}$ & $0.9 \pm 0.4$ & $3.2 \times 10^{-11}$ & $0.9 / 33$ \\
\hline \multicolumn{9}{|l|}{2009} \\
\hline 00031392001 & XRT/PC & 54925.80417 & 54926.00000 & 2.6 & $3.6_{-3.6}^{+6.3}$ & $1.1_{-1.1}^{+1.5}$ & $6.5 \times 10^{-12}$ & $(17.4 / 19)$ \\
\hline $00031392002^{a}$ & XRT/PC & 54973.86319 & 54973.93889 & 2.0 & 3.6 (fixed) & 1.1 (fixed) & $2.0 \times 10^{-12}$ & - \\
\hline $00031392003^{a}$ & XRT/PC & 54974.53819 & 54974.67153 & 1.7 & 3.6 (fixed) & 1.1 (fixed) & $2.2 \times 10^{-12}$ & - \\
\hline $00031392004^{b}$ & XRT/PC & 54978.02153 & 54978.16319 & 1.2 & 3.6 (fixed) & 1.1 (fixed) & $1.3 \times 10^{-12}$ & - \\
\hline \multicolumn{9}{|l|}{2010} \\
\hline 00031854001 & XRT/PC & 55498.62225 & 55498.63470 & 0.99 & $3.7_{-0.7}^{+0.8}$ & $1.1 \pm 0.3$ & $2.6 \times 10^{-10}$ & $1.8 / 52$ \\
\hline \multirow[t]{2}{*}{00031854002} & XRT/WT & 55498.69269 & 55498.88016 & 0.12 & $4.4_{-1.6}^{+2.3}$ & $1.5_{-0.6}^{+0.7}$ & $2.6 \times 10^{-10}$ & $0.8 / 11$ \\
\hline & XRT/PC & & & 2.6 & $3.0_{-0.5}^{+0.0}$ & $1.0 \pm 0.2$ & $2.3 \times 10^{-10}$ & $0.7 / 72$ \\
\hline 00031854003 & XRT/PC & 55500.21293 & 55500.22519 & 0.95 & $10.7_{-4.0}^{-5.0}$ & $0.7 \pm 0.7$ & $6.2 \times 10^{-11}$ & $(46.8 / 50)$ \\
\hline 00031854004 & XRT/PC & 55501.63127 & 55501.64450 & 0.97 & $7.4_{-2.6}^{+3.9}$ & $1.2_{-0.6}^{+0.7}$ & $4.5 \times 10^{-11}$ & $(48.1 / 48)$ \\
\hline 00031854005 & XRT/PC & 55503.63889 & 55503.97292 & 1.3 & $7.0_{-3.4}^{+4.1}$ & $1.0_{-0.7}^{+0.9}$ & $3.0 \times 10^{-11}$ & $(34.8 / 41)$ \\
\hline $00031854006^{a}$ & XRT/PC & 55505.50556 & 55505.71944 & 1.4 & 7.0 (fixed) & 1.0 (fixed) & $8.2 \times 10^{-12}$ & - \\
\hline 00031854007 & XRT/PC & 55507.79097 & 55507.92986 & 1.0 & $4.8_{2.2}^{+2.8}$ & $1.5_{-0.4}^{+0.8}$ & $2.5 \times 10^{-11}$ & $(43.0 / 36)$ \\
\hline 00031854008 & XRT/PC & 55510.45347 & 55510.53889 & 2.4 & $4.7_{-3.6}^{+4.0}$ & $1.1_{-0.9}^{+1.0}$ & $1.5 \times 10^{-11}$ & $(17.0 / 27)$ \\
\hline $00031854009^{a}$ & $\mathrm{XRT} / \mathrm{PC}$ & 55513.46458 & 55513.54931 & 2.2 & 4.7 (fixed) & 1.1 (fixed) & $4.3 \times 10^{-12}$ & - \\
\hline $00031854010^{a}$ & XRT/PC & 55516.56111 & 55516.63472 & 2.3 & 4.7 (fixed) & 1.1 (fixed) & $1.0 \times 10^{-11}$ & - \\
\hline 00031854011 & XRT/PC & 55523.03056 & 55523.10972 & 2.3 & $6.4_{-4.2}^{+4.4}$ & $1.8_{-1.3}^{+1.2}$ & $8.1 \times 10^{-12}$ & $(20.0 / 24)$ \\
\hline 00031854012 & XRT/PC & 55530.05625 & 55530.26944 & 2.8 & $4.8_{-2.1}^{+2.3}$ & $1.2 \pm 0.7$ & $9.3 \times 10^{-12}$ & $(28.0 / 31)$ \\
\hline 00031854013 & XRT/PC & 55537.01806 & 55537.10139 & 2.2 & $8.3_{-1.5}^{+1.1}$ & $2.8_{-1.7}^{+2.1}$ & $3.4 \times 10^{-12}$ & $(13.2 / 11)$ \\
\hline 00031854014 & XRT/PC & 55544.10764 & 55544.99028 & 3.3 & $4.4_{-3.2}^{+3.0}$ & $1.3_{-1.1}^{+1.0}$ & $6.9 \times 10^{-12}$ & $(19.4 / 24)$ \\
\hline 00031854015 & $\mathrm{XRT} / \mathrm{PC}$ & 55550.47847 & 55550.62917 & 3.4 & $4.6_{-2.5}^{+2.9}$ & $1.2_{-0.8}^{+0.9}$ & $5.6 \times 10^{-12}$ & $(11.8 / 21)$ \\
\hline
\end{tabular}

Notes. Spectra extracted from these observations are fitted with an absorbed power law (absorption column density $N_{\mathrm{H}}$ and photon index $\Gamma$ ). $F_{\mathrm{obs}}$ is the absorbed flux in the $1-10 \mathrm{keV}$ energy band. EXP indicates the total exposure time of each observation. ${ }^{(a)}$ We used sostA and wEBPIMMs to estimate this flux; ${ }^{(b)} 90 \%$ c.l. upper limit.

the intercalibration between the Swift/XRT and IBIS/ISGRI instruments and the variability of the source. Both fits gave unacceptable results with $\chi_{\text {red }}^{2}>2.3$. We therefore substituted the power-law component with a cut-off power law (WABs* ${ }^{*}$ CUTOFFPL in XSPEC). This model gave an acceptable fit $\left(\chi_{\text {red }}^{2} /\right.$ d.o.f. $\left.=1.1 / 71\right)$ and we measured a power-law photon index of $\Gamma=0.4 \pm 0.3$, an absorption column density of $N_{\mathrm{H}}=(3.1 \pm 0.7) \times 10^{22} \mathrm{~cm}^{-2}$ and a cut-off energy of $8.0_{-1.0}^{+1.2} \mathrm{keV}$. The normalization constant turned out to be $4.2_{-1.2}^{+1.7}$, which indicates (as expected) that the flux of the source during the Swift observation already decreased with respect to that measured by INTEGRAL during satellite rev. 982 (see also Tables 1 and 2). The unfolded XRT+ISGRI spectrum, the best-fit model, and the residuals from this fit are shown in Fig. 6.

We used the $Z^{2}$-statistic technique (Buccheri et al. 1983; Markwardt et al. 2002) to search for pulsations in all the Swift/XRT observations reported in Table 2. In each of these observations the value of the source spin frequency was estimated accurately by fitting the peak in the $Z^{2}$-statistic periodogram with a model comprising a constant and a sync function. However, because the points in the $Z^{2}$-statistic periodogram are not independent, the error on the position of the centroid of the peak derived in this way could not be considered as a reliable estimate of the uncertainty on the source spin frequency. We thus simulated for each observation 100 event files with the same exposure and number of events as the real file (we assumed a constant flux during each observation). A sinusoidal signal at the frequency determined from the data was introduced artifi-

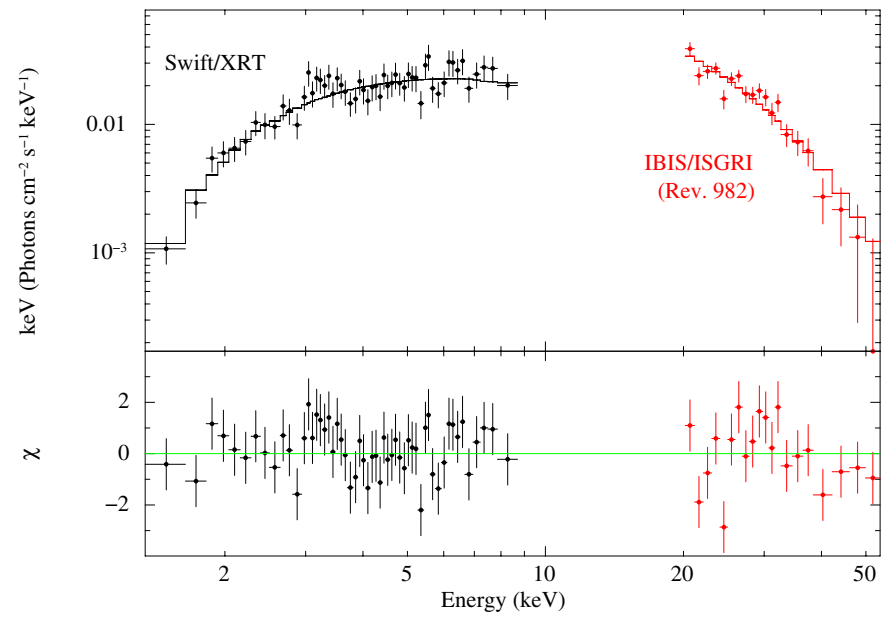

Fig. 6. Unfolded joint Swift/XRT and INTEGRAL/ISGRI spectrum (we used the XRT spectrum extracted from observation ID. 00031854001 and the ISGRI spectrum extracted from the INTEGRAL rev. 982). The best-fit model is obtained with a CUTOFFPL model (see text for details). The residuals from this fit are shown in the bottom panel.

cially and then measured a posteriori by using the $Z^{2}$-statistic (we investigated in each case a total of 1000 frequencies in the range $0.078-0.082 \mathrm{~Hz}$ ). The frequencies with a detection significance $\geq 3 \sigma$ determined with this method were then averaged to 


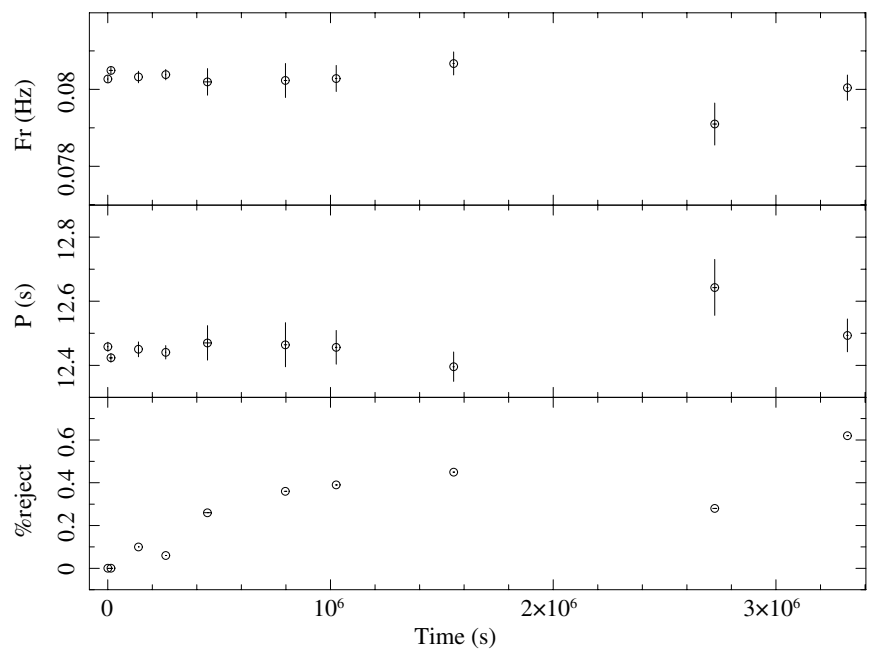

Fig. 7. Results of the search for pulsations in the Swift/XRT data. The upper (middle) panel show the determined frequency (period) of pulsations from the source in each observation as a function of time. In the bottom panel we show the percentage of the total number of simulated lightcurves (100) for each observation that did not result in a statistically significant $(\geq 3 \sigma)$ detection of a spin period at the expected frequency. The reference time $(t=0)$ is 55498.62225 MJD.

derive the value of the best spin frequency for each observation and the associated uncertainty at $1 \sigma$ c.l. (that we assumed equal to 1 standard deviation from the averaged value). We checked for each observation that the frequencies determined from the simulations with this method were all consistent within 1 standard deviation with the best value found by using the $Z^{2}$-statistic on the real event file. The results of this procedure are shown in Fig. 7. In the bottom panel of this figure we also report for each observation the percentage of the 100 simulated lightcurves that did not result in a significant detection of a periodicity $(\leq 3 \sigma)$. The observations characterized by a lower source flux or a shorter exposure gave, as expected, a higher percentage of failed detections. This suggests that the percentage reported in the figure can be considered a reliable estimate of the detection significance of the periodicity in each observation.

The results reported in Fig. 7 show that owing to the relative large uncertainties that affect most of the measurements, no significant variations of the source spin frequency could be measured during the Swift follow-up observations. This prevented any even tentative determination of an orbital solution for IGR J19294+1816 through the pulse arrival time technique.

To search for a confirmation of the suggested $\sim 117 \mathrm{~d}$ orbital period of IGR J19294+1816 (see Sect. 1), we therefore adopted an alternative method. We obtained the long-term lightcurve of the source from the Swift/BAT survey web-page ${ }^{4}$ binned snapshot-by-snapshot. All photon arrival times were corrected to the solar system barycenter with the task EARTH2sun. Because the $Z^{2}$-statistic technique is particularly suited for un-binned event files rather than for lightcurves, we searched for periodicities in the BAT data by using the Lomb-Scargle (LS) periodogram (we adopted the fast algorithm described in Press \& Rybicki 1989, and scanned periods above 31 days with an oversampling factor of 8). Because the uncertainties affecting the Swift/BAT data change significantly from pointing to pointing because of the different exposure time and off-axis position of the source with respect to the instrument aim-point, we also implemented the

\footnotetext{
${ }^{4}$ http://heasarc.gsfc.nasa.gov/docs/swift/results/ bs $58 \mathrm{mon} /$.
}

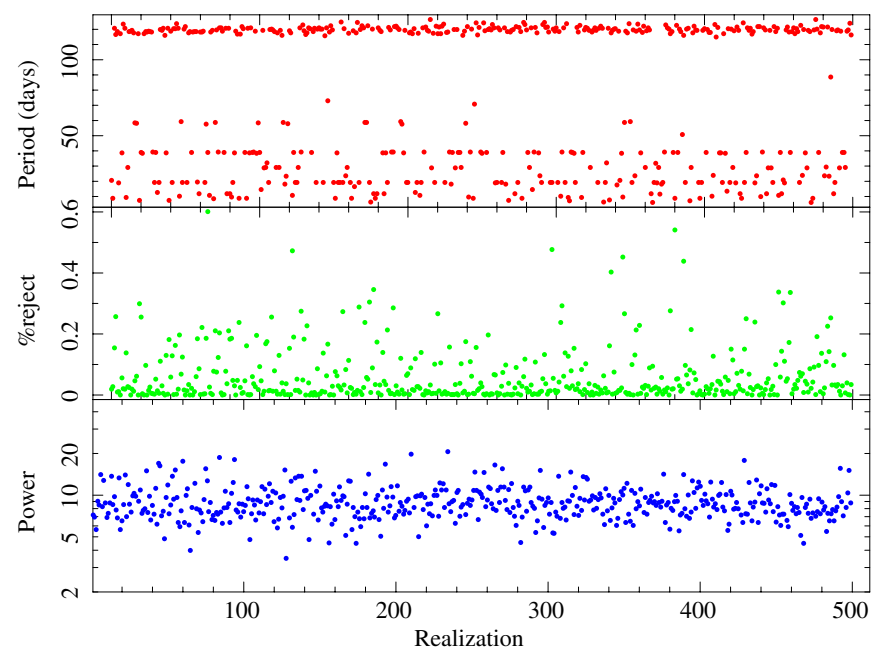

Fig. 8. Results of the simulations carried out to investigate the significance of the period at $\sim 120$ days found from the Swift/BAT data. The upper panel shows the value of the period for each simulation (the number of the simulation is reported on the $\mathrm{x}$-axis). The middle panel reports the probability that the detection of a period is false in each simulation (i.e., a value of 0.1 corresponds to a detection significance of $90 \%$ c.1.), and the lower panel the power of each peak in the corresponding LS diagram.

weighting scheme of the BAT lightcurve proposed by Corbet et al. (2007, in our case the correction factor $V_{S}$ introduced to account for the source variability turned out to be negative and thus was set to zero). The Lomb-Scargle periodogram was realized for both the original Swift/BAT lightcurve and for the 500 lightcurves simulated from it. In performing the simulation, we assumed for each lightcurve a duration equal to that of the true Swift/BAT lightcurve and drew the source countrate in each time bin from a normal distribution centered on the real measured count-rate ${ }^{5}$. We set the corresponding standard deviation equal to the measured error on the count-rate. The results of this analysis are reported in Fig. 8. The histogram of the periods determined from the simulated data showed a single prominent peak at $\sim 120$ days, which thus turned out to be the most probable period (the simulation does not assume a priori a periodicity). However, we noticed that only 266 out of the 500 simulated lightcurves displayed a peak at a period of $\sim 120$ days, and from these we estimated the best orbital period at $P_{\text {orb }}=120 \pm 2$ days $(68 \%$ c.l.). Most of the remaining lightcurves (209) show a prominent peak at frequencies close to higher harmonics of the fundamental frequency. This distribution of the power among several harmonics would be expected according to the findings of Corbet \& Krimm (2009), who showed that the folded lightcurve of the source was strongly not sinusoidal.

To refine our determination of the orbital period, we also looked for periodicities in the Swift/BAT lightcurve by exploiting the epoch-folding technique described by D'Aì et al. (2011). We folded the light-curve in 16 phase bins by using periods in the range $15-600 \mathrm{~d}$. The sampling in period was chosen to be 3 times finer than the resolution of the method, i.e. $\Delta v=P^{2} /\left(N \Delta_{\mathrm{BAT}}\right)$ (here, $P$ is the period, $N$ is the number of phase bins, and $\Delta_{\mathrm{BAT}}$ is the data span length; Buccheri et al. 1983). We then computed for each of the folded lightcurves the corresponding $\chi^{2}$ and

5 This assumption is justified for a coded mask instrument, because the source signal typically constitutes only a few percent of the large number of counts that characterize the background. 

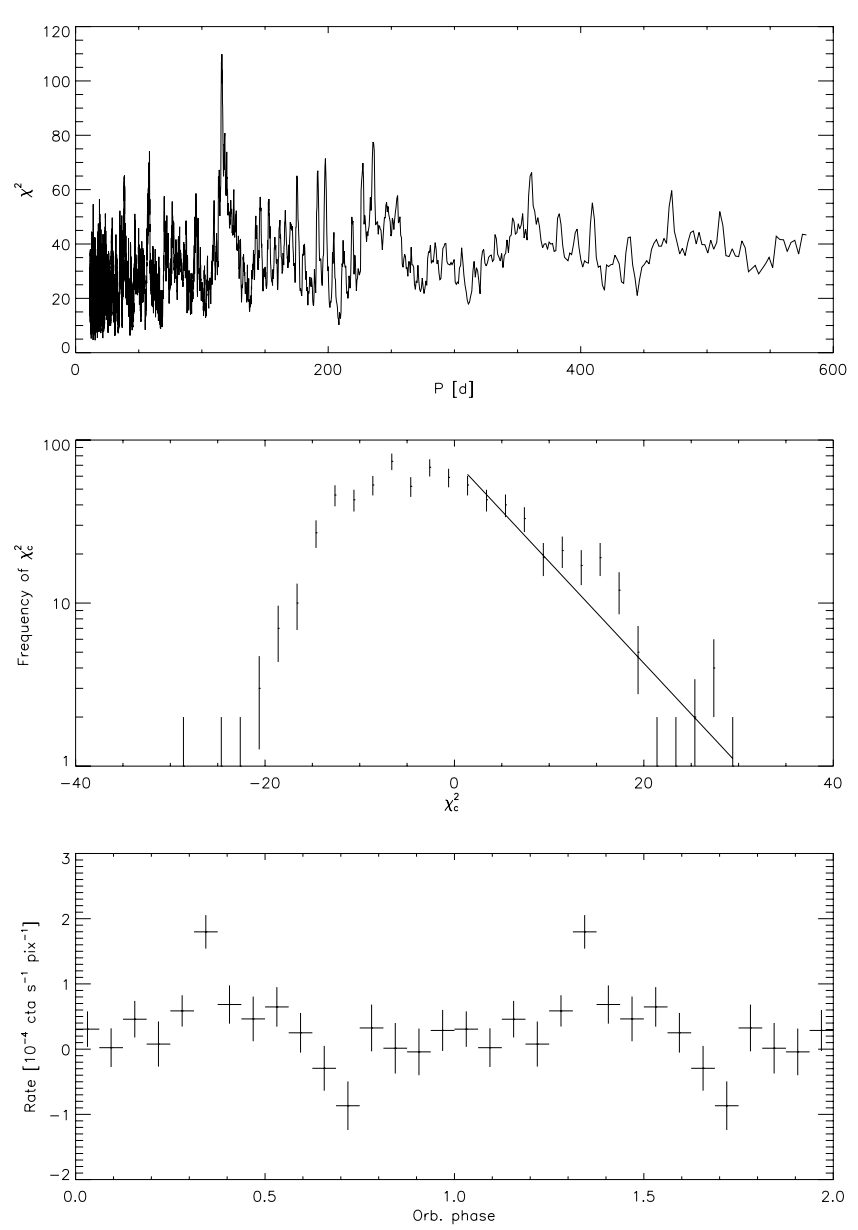

Fig. 9. Results of the search for periodicity in the Swift/BAT data obtained with the technique described by D'Aì et al. (2011). The upper panel shows the $\chi^{2}$ computed from the Swift/BAT lightcurve folded at different periods in the range 15-600 days. The most prominent peak is at $116.2 \pm 0.6$ days. To estimate the significance of this peak, we followed the procedure described in D'Aì et al. (2011), and report the histogram of the detrended $\chi^{2}$ values in the middle panel. We show in the bottom panel the Swift/BAT lightcurve $(15-50 \mathrm{keV})$ of the source folded at the best determined orbital period (116.2 \pm 0.6 days). The reference time used is 54877.3957 MJD. Each phase bin in the plot corresponds to an integration time of three days.

found the most prominent excess at a period of $116.2 \pm 0.6$ days. The estimated chance probability that this detection would correspond to a spurious effect is about $1 \%$ (see Fig. 9). In the bottom panel of Fig. 9 we also show the Swift/BAT lightcurve folded at the best determined spin period. The reference epoch was chosen at 54877.3957 MJD, and we notice the presence of a prominent peak in the source count rate at phase $0.34 \pm 0.03$. Considering an orbital period of $116.2 \pm 0.6$ days, the epoch of the peak would agree with the prediction of Corbet \& Krimm (2009).

We conclude that further observations are probably needed to firmly establish and eventually refine the orbital period of the source.

\section{Discussion and conclusions}

We reported on the INTEGRAL and Swift observations carried out for about two months after the newly discovered outburst of IGR J19294+1816 on 2010 October 28 (Bozzo et al.
2010). During these observations, IGR J19294+1816 displayed a smoothly decreasing X-ray flux with a decay time-scale similar to that observed during the outburst in 2009. Pulsations were clearly detected in the INTEGRAL data up to $\sim 50 \mathrm{keV}$ and in the Swift/XRT data. Owing to the relatively low X-ray flux of the source during most of the XRT observations, the accuracy with which we could measure its spin period was far too low to determine an orbital solution for IGR J19294+1816 with the pulse arrival times technique. Instead, we found a marginal detection of this periodicity with both the LS periodogram and the epoch-folding technique. We found the best orbital period at $116.2 \pm 0.6$ days, with a chance probability of $1 \%$ to be a spurious detection.

As Rodriguez et al. (2009a) remarked, the spin and orbital period of IGR J19294+1816 (if confirmed) would nicely place the source in the part of the $P_{\text {spin }}-P_{\text {orb }}$ diagram (Corbet 1986) populated by the so-called Be X-ray binaries. The INTEGRAL and Swift observations of the source carried out during the outburst in 2010 provide further support to this hypothesis. The $\mathrm{X}$-ray pulsations at $\sim 12.5 \mathrm{~s}$ and the high rms fractional amplitude measured up to $50 \mathrm{keV}$ are typical of Be X-ray binaries and are not usually observed in the SFXTs (the $\sim 6$ days long outburst shown in Fig. 5 would also be typical for a Be X-ray binary; see e.g., Ziolkowski 2002; Reig 2011, for recent reviews). Among all the candidate and confirmed sources in the SFXT class ( 15 objects), so far only ${ }^{6}$ the intermediate SFXT IGR J18483-0311 showed pulsations with a period similar to that of IGR J19294+1816 ( 21 s detected up to $\sim 40 \mathrm{keV}$, Sguera et al. 2007). However, the smooth and gradual decline of the X-ray flux from IGR J19294+1816 during two months spanned by the Swift observations seems to be different from the behavior displayed by the SFXT sources. Indeed, these sources usually show a rapid (thousands of seconds) decline of the X-ray flux of 3-5 orders of magnitude after the occurrence of an outburst. In those cases in which a detailed monitoring could be carried out with Swift/XRT after the occurrence of a bright event, it was also noticed that a pronounced variability in the X-ray flux (a factor $\sim 100$ ), accompanied by significant changes in the spectral parameters (e.g., the absorption column density), characterize the source emission during the entire orbit (see e.g., Bozzo et al. 2008, 2009; Sidoli et al. 2009). The slow decline of the X-ray flux from IGR J19294+1816, combined with the fairly small changes in the spectral parameters across the entire Swift monitoring, suggest that the behavior of IGR J19294+1816 is closer to that of the Be X-ray binaries. We thus conclude that IGR J19294+1816 is most likely a Be X-ray binary and not an SFXT.

Acknowledgements. We thank the Swift team for the prompt scheduling of all the follow-up observations of IGR J19294+1816, and an anonymous referee for useful comments. This research has made use of the XRT Data Analysis Software (XRTDAS) developed under the responsibility of the ASI Science Data Center (ASDC), Italy.

\section{References}

Bozzo, E., Falanga, M., \& Stella, L. 2008, ApJ, 683, 1031

Bozzo, E., Giunta, A., Stella, L., et al. 2009, A\&A, 502, 21

Bozzo, E., Rodriguez, J., Wilms, J., et al. 2010, The Astronomer's Telegram, 2983, 1

Bozzo, E., Giunta, A., Cusumano, G., et al. 2011, A\&A, submitted Buccheri, R., Bennett, K., Bignami, G. F., et al. 1983, A\&A, 128, 245

${ }^{6}$ Note that Bozzo et al. (2011) showed that the NS hosted in the SFXT IGR J18410-0535 might not be pulsating at $\sim 4.7 \mathrm{~s}$ as it was previously reported. 


\section{E. Bozzo et al.: IGR J19294+1816 in outburst}

Burrows, D. N., Hill, J. E., Nousek, J. A., et al. 2005, Space Sci. Rev., 120, 165 Corbet, R. H. D. 1986, MNRAS, 220, 1047

Corbet, R. H. D., \& Krimm, H. A. 2009, The Astronomer's Telegram, 2008, 1

Corbet, R. H. D., Markwardt, C. B., \& Tueller, J. 2007, ApJ, 655, 458

Courvoisier, T., Walter, R., Beckmann, V., et al. 2003, A\&A, 411, L53

D’ Aì, A., La Parola, V., Cusumano, G., et al. 2011, A\&A, 529, A30

Ferrigno, C., Segreto, A., Santangelo, A., et al. 2007, A\&A, 462, 995

Jenke, P., Camero-Arranz, A., Finger, M. H., \& Caballero, I. 2010, The Astronomer's Telegram, 2985, 1

Krimm, H. A., Barthelmy, S. D., Baumgartner, W., et al. 2009, The Astronomer's Telegram, 1999, 1

Lebrun, F., Leray, J. P., Lavocat, P., et al. 2003, A\&A, 411, L141

Lund, N., Budtz-Jørgensen, C., Westergaard, N. J., et al. 2003, A\&A, 411, L231

Markwardt, C. B., Swank, J. H., Strohmayer, T. E., in 't Zand, J. J. M., \&

Marshall, F. E. 2002, ApJ, 575, L21

Press, W. H., \& Rybicki, G. B. 1989, ApJ, 338, 277
Reig, P. 2011, Ap\&SS, 332, 1

Rodriguez, J., Tomsick, J. A., Bodaghee, A., et al. 2009a, A\&A, 508, 889

Rodriguez, J., Tuerler, M., Chaty, S., \& Tomsick, J. A. 2009b, The Astronomer's Telegram, 1998, 1

Segreto, A., \& Ferrigno, C. 2007, in ESA Spec. Pub., 622, 633

Sguera, V., Hill, A. B., Bird, A. J., et al. 2007, A\&A, 467, 249

Sidoli, L., Romano, P., Ducci, L., et al. 2009, MNRAS, 397, 1528

Strohmayer, T., Rodriquez, J., Markwardt, C., et al. 2009, The Astronomer's Telegram, 2002, 1

Turler, M., Rodriguez, J., \& Ferrigno, C. 2009, The Astronomer's Telegram, 1997, 1

Ubertini, P., Lebrun, F., Di Cocco, G., et al. 2003, A\&A, 411, L131

van der Klis, M. 1988, in Timing Neutron Stars, ed. H. Ogelman, \& E. P. J. van den Heuvel, NATO ASI Series C (Dordrecht: Kluwer), 262, 27

Walter, R., \& Zurita Heras, J. 2007, A\&A, 476, 335

Ziolkowski, J. 2002, Mem. Soc. Astron. Ital., 73, 1038 